\title{
A Crystal Plasticity Model with Irradiation Effect for the Mechanical Behavior of FCC Metals
}

\author{
Junfeng Nie ${ }^{1 \star} \quad$ Yunpeng Liu $^{1} \quad$ Pandong Lin $^{1} \quad$ Qihao Xie $^{3} \quad$ Zhanli Liu $^{2}$ \\ $\left({ }^{1}\right.$ Institute of Nuclear and New Energy Technology, Collaborative Innovation Center of Advanced Nuclear \\ Energy Technology, Key Laboratory of Advanced Reactor Engineering and Safety of Ministry of Education, \\ Tsinghua University, Beijing 100084, China) \\ $\left({ }^{2}\right.$ Applied Mechanics Lab., School of Aerospace Engineering, Tsinghua University, Beijing 100084, China) \\ $\left({ }^{3}\right.$ Data Science and Information Technology Research Center, Tsinghua-Berkeley Shenzhen Institute, \\ Shenzhen 518055, China)
}

Received 28 February 2019; revision received 19 September 2019; Accepted 24 September 2019; published online 12 October 2019

(C) The Author(s) 2019

\begin{abstract}
In this paper, a crystal plasticity model considering the irradiation effect based on the thermal activation theory is established. The evolutions of screw dislocations, edge dislocations, and stacking fault tetrahedrals (SFTs) (induced by irradiation) are included into the model. The interactions between dislocations and irradiation-induced SFTs are also considered. The constitutive model is numerically implemented on the ABAQUS platform through UMAT subroutine and applied to study the irradiation effect on the mechanical behavior of pure copper. The mechanical properties of single and polycrystalline copper are studied, and the simulation results show that the constitutive model can properly predict the mechanical behavior of irradiated pure copper. Especially for polycrystalline copper, the simulation results are in good agreement with the experimental data.
\end{abstract}

KEY WORDS Crystal plasticity, Irradiation effect, Uniaxial tension, Dislocation, FCC crystal

\section{Introduction}

In nuclear industry, the mechanical properties of metal materials are influenced by irradiation and temperature. After irradiation, metal materials with different crystal structures always show an irradiation effect, i.e., a significant increase in yield strength and a decrease in toughness $[1,2]$. The irradiation effect becomes more pronounced as the irradiation dose increases. When the irradiation dose increases to a certain value, an upper yield point will appear and then the stress decreases. Moreover, the intensity of irradiation effect is also affected by the irradiation temperature. The results of the post-irradiation tensile testing exhibit stronger irradiation hardening and show that materials irradiated at room temperature have less ductility compared with the high temperature's occasion $[3,4]$.

From the microperspective, materials in the irradiation environment are impacted by energetic particles, which incurs cascade reaction subsequently and results in a large number of nanoscale irradiation defects, such as vacancies, interstitial atoms, voids, precipitates, stacking fault tetrahedrals (SFTs),

\footnotetext{
* Corresponding author. E-mail: niejf@tsinghua.edu.cn
} 
small dislocation loops, and so on [5]. The type and density of defect are related to the crystal structure. For example, Singh et al. [6] investigated the microstructure and associated tensile properties of irradiated FCC (Cu, Pd and 304 stainless steel) and BCC (Fe, Mo) metals by experiments, and revealed the differences and similarities of metals with different crystal structures after irradiation.

Otherwise, many studies show that the plastic deformation of the crystal is attributed to the dislocations slipping along the corresponding slip planes. Crystal plasticity theory is a basic theory for describing the plastic deformation at mesoscale, which connects the microscopic slip mechanism with the macroscopic plastic deformation behavior [7-10]. The deformation mechanism of the irradiated FCC materials is greatly affected by the dislocations and irradiation defects. Cheong et al. [11] developed a dislocation-mechanics-based crystallographic theory to study the mechanical properties of thin polycrystalline $\mathrm{Cu}$ specimens. Though the irradiation effect has not been considered, the evolution model of screw and edge dislocations is useful for studying the mechanical behavior of FCC crystal. Arsenlis et al. [12] introduced an internal state variable model for the mechanical behavior of irradiated structural materials within a multi-scale framework. Using the model, the behavior of $\mathrm{Cu}$ tensile specimens with varying irradiation damage was simulated. De et al. [13] proposed a defect- and dislocation-density-based evolution model to capture the features of irradiation hardening as well as intra-granular softening. The Jacobian-free multi-scale method (JFMM) was further applied to improve the computational performance in the polycrystalline aggregate simulations by using a Newton-Krylov process. The mechanical responses of neutron-irradiated single and polycrystalline OFHC copper were studied and the model could capture experimentally observed grain-level phenomena. Chen et al. [14] investigated the mechanical behavior and crystallographic texture evolution of irradiated FCC metals based on a physical theoretical model. The study revealed the texture evolution along with different orientations before and after irradiation. It was concluded that irradiation-induced defects could affect the mechanical behavior and texture evolution of metals, both of which were closely related to irradiation hardening. Xiao et al. [15] established a tensorial crystal plasticity model with both irradiation and temperature effects. Using the model, the mechanical behavior and characteristics of irradiated polycrystals at different temperatures could be captured. Besides, Xiao et al. [16] also explored the effects of irradiation damage and crystal size on the mechanical behavior of FCC single crystals based on a unified size-dependent tensorial plasticity model. Krishna et al. [17] proposed a micromechanicsbased model for copper subjected to neutron irradiation. The defect evolution was considered in the model to reflect the prevention and annihilation effects of defects on dislocations.

In this paper, a crystal plasticity model considering irradiation effect is established by tracking the evolutions of screw dislocations, edge dislocations, and SFTs induced by the irradiation. The model can also reflect the interactions between dislocations and irradiation defects and has been implemented numerically on the ABAQUS finite element platform. Using the model, the mechanical behaviors of single and polycrystalline copper before and after irradiation are studied and predicted.

\subsection{Crystal Plasticity Theory}

\section{Constitutive Model}

According to the classical crystal plasticity theory [7-10], the total deformation gradient $\boldsymbol{F}$ is divided into two parts: $\boldsymbol{F}^{\mathrm{P}}$, which denotes crystallographic slip, and $\boldsymbol{F}^{*}$, which represents stretching and rotation of the lattice.

$$
\begin{gathered}
\boldsymbol{F}^{\alpha}=\boldsymbol{F}^{*} \boldsymbol{F}^{\mathrm{P}} \\
\boldsymbol{F}^{\mathrm{P}}=\sum_{\alpha=1}^{N}\left(\boldsymbol{I}+\gamma^{\alpha} \boldsymbol{m}^{\alpha} \otimes \boldsymbol{n}^{\alpha}\right)
\end{gathered}
$$

where $N$ is the number of all activated slip systems, $\boldsymbol{I}$ is the second-order identical tensor, $\gamma^{\alpha}$ is the shear strain. $\boldsymbol{m}^{\alpha}$ and $\boldsymbol{n}^{\alpha}$ are the slip direction and normal-to-slip plane of the $\alpha$ slip system, respectively. The slip direction and normal-to-slip plane after deformation are given by

$$
\boldsymbol{m}^{* \alpha}=\boldsymbol{F}^{*} \boldsymbol{m}^{\alpha}
$$




$$
\boldsymbol{n}^{* \alpha}=\boldsymbol{n}^{\alpha} \boldsymbol{F}^{*-1}
$$

The velocity gradient $\boldsymbol{L}$ can be decomposed into the lattice part $\boldsymbol{L}^{*}$ and the plastic part $\boldsymbol{L}^{\mathrm{P}}$ :

$$
\begin{gathered}
\boldsymbol{L}=\dot{\boldsymbol{F}} \boldsymbol{F}^{-1}=\boldsymbol{L}^{*}+\boldsymbol{L}^{\mathrm{P}} \\
\boldsymbol{L}^{*}=\dot{\boldsymbol{F}}^{*} \boldsymbol{F}^{*-1} \\
\boldsymbol{L}^{\mathrm{P}}=\boldsymbol{F}^{*} \dot{\boldsymbol{F}}^{\mathrm{P}} \boldsymbol{F}^{\mathrm{P}-1} \boldsymbol{F}^{*-1}
\end{gathered}
$$

$\boldsymbol{L}$ can be also decomposed into the deformation rate tensor $\boldsymbol{D}$ and the spin rate tensor $\boldsymbol{\Omega}$.

$$
\begin{gathered}
\boldsymbol{L}=\boldsymbol{D}+\boldsymbol{\Omega}=\frac{1}{2}\left(\boldsymbol{L}+\boldsymbol{L}^{\mathrm{T}}\right)+\frac{1}{2}\left(\boldsymbol{L}-\boldsymbol{L}^{\mathrm{T}}\right) \\
\boldsymbol{D}=\boldsymbol{D}^{*}+\boldsymbol{D}^{\mathrm{P}} \\
\boldsymbol{\Omega}=\boldsymbol{\Omega}^{*}+\boldsymbol{\Omega}^{\mathrm{P}} \\
\boldsymbol{D}^{\mathrm{P}}=\frac{1}{2}\left(\boldsymbol{L}^{\mathrm{P}}+\boldsymbol{L}^{\mathrm{PT}}\right)=\sum_{\alpha=1}^{N} \boldsymbol{\mu}^{\alpha} \dot{\gamma}^{\alpha} \\
\boldsymbol{\mu}^{\alpha}=\frac{1}{2}\left(\boldsymbol{m}^{* \alpha} \otimes \boldsymbol{n}^{* \alpha}+\boldsymbol{n}^{*^{\alpha}} \otimes \boldsymbol{m}^{* \alpha}\right) \\
\boldsymbol{\Omega}^{\mathrm{P}}=\frac{1}{2}\left(\boldsymbol{L}^{\mathrm{P}}-\boldsymbol{L}^{\mathrm{PT}}\right)=\sum_{\alpha=1}^{N} \boldsymbol{W}^{\alpha} \dot{\gamma}^{\alpha} \\
\boldsymbol{W}^{\alpha}=\frac{1}{2}\left(\boldsymbol{m}^{* \alpha} \otimes \boldsymbol{n}^{* \alpha}-\boldsymbol{n}^{* \alpha} \otimes \boldsymbol{m}^{* \alpha}\right)
\end{gathered}
$$

where $\dot{\gamma}^{\alpha}$ is the slipping rate of the $\alpha$ slip system.

The crystal macroscopic deformation and microslipping rate are portrayed through the above formulas. It is assumed that $\hat{\tau}$ is the Jaumann derivative of Kirchhoff stress $\boldsymbol{\tau}$ which takes the initial configuration as the reference state. The relationship between the shear stress and the shear strain can be derived by

$$
\begin{gathered}
\hat{\boldsymbol{\tau}}=\boldsymbol{C}: \boldsymbol{D}-\sum_{\alpha=1}^{N}\left(\boldsymbol{C}: \boldsymbol{\mu}^{\alpha}+\boldsymbol{\beta}^{\alpha}\right) \dot{\gamma}^{\alpha} \\
\boldsymbol{\beta}^{\alpha}=\boldsymbol{W}^{\alpha} \boldsymbol{\tau}-\boldsymbol{\tau} \boldsymbol{W}^{\alpha} \\
\hat{\boldsymbol{\tau}}=\dot{\boldsymbol{\tau}}-\boldsymbol{\Omega} \boldsymbol{\tau}+\boldsymbol{\tau} \boldsymbol{\Omega} \\
\tau^{\alpha}=\boldsymbol{\tau}: \boldsymbol{\mu}^{\alpha}
\end{gathered}
$$


where $C$ is the stiffness tensor. According to the work of Kocks [18], the slipping rate of the $\alpha$ slip system can be expressed as

$$
\begin{gathered}
\left\{\begin{array}{l}
\dot{\gamma}^{\alpha}=0 \\
\dot{\gamma}^{\alpha}=\dot{\gamma}_{0}^{\alpha} \exp \left\{-\frac{Q_{0}}{k T}\left[1-\left(\frac{\left|\tau^{\alpha}\right|-g^{\alpha}}{\widehat{\tau}^{\alpha}}\right)^{p}\right]^{q}\right\} \operatorname{sgn}\left(\tau^{\alpha}\right) \\
\left(\left|\tau^{\alpha}\right|>g^{\alpha}\right)
\end{array}\right. \\
\widehat{\tau}^{\alpha}=\widehat{\tau}_{0}^{\alpha} \frac{G}{G_{0}}
\end{gathered}
$$

where $Q_{0}$ denotes the activation energy required to overcome the obstacles to dislocation motion, $p$ and $q$ are flow-rule-related parameters, $G$ and $G_{0}$ represent the shear modulus at temperature $T$ and $0 \mathrm{~K}$, respectively. $\widehat{\tau}^{\alpha}$ and $\widehat{\tau}_{0}^{\alpha}$ represent the lattice friction stress of each slip system at temperature $T$ and $0 \mathrm{~K}$, respectively. It is assumed that $\widehat{\tau}_{0}^{\alpha}$ is the same in each sliding system, so $\widehat{\tau}_{0}^{\alpha}$ is simplified to $\widehat{\tau}_{0}$. And the term $g^{\alpha}$ represents the total slip resistance to dislocation motion, including the parts caused by dislocations and irradiation defects.

\subsection{Evolution of Dislocations}

The strain hardening behavior of the crystal is closely related to the dislocation density. The dislocation density increases gradually during the process of plastic deformation, making the interaction between dislocations more intense. So the dislocation motion becomes more difficult. The strain hardening mechanisms include: dislocation congestion, dislocation intersection, the formation of fixed dislocations, the reduction of Frank-Lead dislocation sources, and so on. In order to describe the strain hardening behavior of the crystal, Cheong [11] proposed a new hardening formula based on the dislocation density,

$$
\begin{gathered}
g^{\alpha}=\lambda G b^{\alpha} \sqrt{\sum_{\beta=1}^{N}\left[h^{\alpha \beta}\left(\rho_{\mathrm{e}}^{\beta}+\rho_{\mathrm{s}}^{\beta}\right)\right]} \\
h^{\alpha \beta}=\omega_{1}+\left(1-\omega_{2}\right) \delta^{\alpha \beta}
\end{gathered}
$$

here $\lambda$ is a statistical coefficient, $b^{\alpha}$ is the magnitude of the Burgers vector. $\omega_{1}$ and $\omega_{2}$ are the self and latent hardening moduli, respectively. $\delta^{\alpha \beta}$ is the Kronecker delta. $\rho_{\mathrm{e}}^{\alpha}$ and $\rho_{\mathrm{s}}^{\alpha}$ are the densities of edge and screw dislocations, respectively.

It is assumed that dislocation multiplication is associated with the expansion of dislocation loops from Frank-Read-type sources and dislocation reduction is associated with the annihilation reaction when the anti-parallel dislocations meet during the motion process. According to the study of Cheong [11], the evolutions of edge dislocations and screw dislocations can be expressed as

$$
\begin{gathered}
\dot{\rho}_{\mathrm{e}}^{\alpha}=\frac{C_{\mathrm{e}}}{b^{\alpha}}\left(K_{\mathrm{e}} \sqrt{\sum_{\beta=1}^{N} \rho_{\mathrm{t}}^{\beta}}-2 d_{\mathrm{e}} \rho_{\mathrm{e}}^{\alpha}\right)\left|\dot{\gamma}^{\alpha}\right| \\
\dot{\rho}_{\mathrm{s}}^{\alpha}=\frac{C_{\mathrm{s}}}{b^{\alpha}}\left[K_{\mathrm{s}} \sqrt{\sum_{\beta=1}^{N} \rho_{\mathrm{t}}^{\beta}}-\left(\pi d_{\mathrm{s}}^{2} K_{\mathrm{s}} \sqrt{\sum_{\beta=1}^{N} \rho_{\mathrm{t}}^{\beta}}+2 d_{\mathrm{s}}\right) \rho_{\mathrm{s}}^{\alpha}\right]\left|\dot{\gamma}^{\alpha}\right|
\end{gathered}
$$

The terms $C_{\mathrm{e}}$ and $C_{\mathrm{s}}$ characterize the contributions of edge and screw dislocations to the slip rate, respectively. $d_{\mathrm{s}}$ and $d_{\mathrm{e}}$ are the critical edge annihilation distances. $\rho_{\mathrm{t}}$ is the total density of dislocations in a slip system. $K_{\mathrm{e}}$ and $K_{\mathrm{s}}$ are dimensionless constants, both of which control the motion of dislocations. 


\subsection{Evolution of Defects}

Irradiation defects in the FCC copper crystal are mainly SFTs $[4,5]$, with a low density of small dislocation loops. In this paper, the defect evolution model proposed by Krishna [17] is adopted where the SFT is regarded as the main irradiation defect. Besides, the annihilation of irradiation defects is due to the reactions between dislocations and SFTs. The full absorption of SFTs by screw dislocations and partial absorption of SFTs by edge dislocations are reported in many studies [19-21]. The absorption mechanism is simplified and then applied to the model. However, other mechanisms that lead to annihilation, such as localized plastic heating, or stress-assisted conversion from SFTs into Frank loops, have not been considered at present.

It is assumed that the defects within the critical distance are readily absorbed. The annihilation mechanism of a defect encountering a screw dislocation is shown in Fig. 1. The small triangle represents an SFT. As shown in Fig. 1, the dislocation with a length of $L_{\mathrm{s}}^{\alpha}$ moves a distance of $Y_{\mathrm{s}}^{\alpha}$ before arrested by forest dislocations in a time interval $d t$. So the annihilation area of the defects is

$$
A_{\mathrm{s}}^{\alpha}=\left(2 d_{\mathrm{q}} Y_{\mathrm{s}}^{\alpha}+\pi d_{\mathrm{q}}^{2}\right)
$$

here $d_{\mathrm{q}}$ is the critical distance.

According to the similar expression obtained for edge dislocations, the additional term $\pi d_{\mathrm{q}}^{2}$ is deleted due to its inability to cross-slip. In this case, the annihilation area of the defects is

$$
A_{\mathrm{e}}^{\alpha}=2 d_{\mathrm{q}} Y_{\mathrm{e}}^{\alpha}
$$

here $Y^{\alpha}$ is the mean free path of the dislocation segment, which is defined as the moving distance of the segment before arrested by forest dislocations $\left(Y_{\mathrm{e}}^{\alpha}\right.$ is for edge dislocation and $Y_{\mathrm{s}}^{\alpha}$ is for screw dislocation).

$$
\begin{gathered}
Y_{\mathrm{e}}^{\alpha}=\frac{l_{\mathrm{m}}^{\alpha}}{K_{\mathrm{e}}} \\
Y_{\mathrm{s}}^{\alpha}=\frac{l_{\mathrm{m}}^{\alpha}}{K_{\mathrm{s}}}
\end{gathered}
$$

$l_{\mathrm{s}}$ is the mean obstacle spacing. According to the geometric considerations, $l_{\mathrm{m}}$ can be expressed as

$$
l_{\mathrm{m}}^{\alpha}=\frac{1}{\sqrt{\sum_{\beta=1}^{N} \rho_{\mathrm{t}}^{\beta}}}
$$

Then the annihilation probabilities of SFTs are

$$
\begin{aligned}
& P_{\mathrm{e}, \mathrm{ann}}=\frac{A_{\mathrm{e}}^{\alpha} L_{\mathrm{e}}^{\alpha}}{V}=A_{\mathrm{e}}^{\alpha} \rho_{\mathrm{e}}^{\alpha} \\
& P_{\mathrm{s}, \mathrm{ann}}=\frac{A_{\mathrm{s}}^{\alpha} L_{\mathrm{s}}^{\alpha}}{V}=A_{\mathrm{s}}^{\alpha} \rho_{\mathrm{s}}^{\alpha}
\end{aligned}
$$

Therefore, the change rate of the SFT density is

$$
\dot{\rho}_{\mathrm{def}}^{\alpha}=-\left(A_{\mathrm{e}}^{\alpha} \rho_{\mathrm{e}}^{\alpha}+A_{\mathrm{s}}^{\alpha} \rho_{\mathrm{s}}^{\alpha}\right) \varphi \rho_{\mathrm{def}}^{\alpha} d_{\mathrm{def}}^{\alpha} \frac{\left|\dot{\gamma}^{\alpha}\right|}{b^{\alpha}}
$$

here $\rho_{\text {def }}^{\alpha}$ is the areal density of the irradiation defects in the $\alpha$ slip system. $d_{\text {def }}^{\alpha}$ is the average defect size. It is supposed that only the overlapped or truncated SFTs are annihilated, which account for about $70 \%-80 \%$ of the total defects $[22,23]$ and the proportion is represented by the term $\varphi$. The evolution of defects is associated with the shear strain rate through Eq. (32).

Similar to the forest dislocations, SFTs and other defects can impede the dislocation motion, resulting in the irradiation hardening. In order to simulate the mechanical behavior of the irradiated crystal 


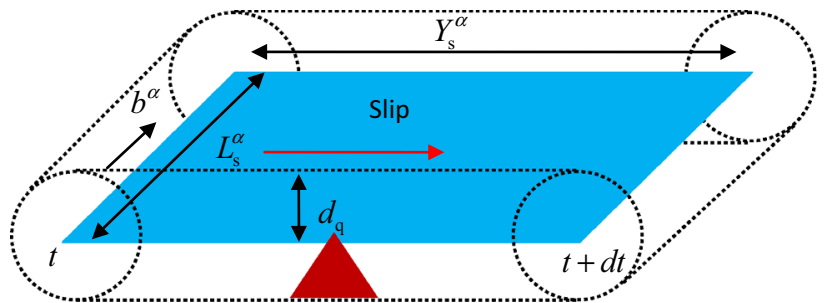

Fig. 1. Schematic of reactions between dislocations and other defects

material, an irradiation hardening term is introduced into Eq. (21) and the irradiated slip resistance is

$$
\begin{gathered}
g^{\alpha}=\lambda G b^{\alpha} \sqrt{\sum_{\beta=1}^{N}\left[h^{\alpha \beta}\left(\rho_{\mathrm{e}}^{\beta}+\rho_{\mathrm{s}}^{\beta}\right)\right]+\sum_{\beta=1}^{N} i^{\alpha \beta} \rho_{\mathrm{def}}^{\beta}} \\
i^{\alpha \beta}=\omega_{\mathrm{i} 1}+\left(1-\omega_{\mathrm{i} 2}\right) \delta^{\alpha \beta}
\end{gathered}
$$

here $i^{\alpha \beta}$ is the coefficient representing the interactions between defects and dislocations in different slip systems. $\omega_{\mathrm{i} 1}$ and $\omega_{\mathrm{i} 2}$ are the self and latent hardening moduli, respectively. The areal initial density of SFTs can be calculated by

$$
\rho_{\text {def }}^{\alpha}=N_{\text {def }}^{\alpha} d_{\text {def }}
$$

where $N_{\text {def }}^{\alpha}$ is the volume density of SFTs.

\section{Finite Element Method}

The finite element platform ABAQUS provides an interface for users to define a constitutive model in a Fortran subroutine called UMAT [24]. In this paper, the tangent modulus method developed by Pierce [25] is used to implement the UMAT. It is defined that $\Delta t$ is the time increment. The increment of shear strain in slip system $\alpha$ can be expressed as

$$
\Delta \gamma^{\alpha}=\gamma^{\alpha}(t+\Delta t)-\gamma^{\alpha}(t)
$$

Then a linear interpolation within $\Delta t$ is employed as:

$$
\Delta \gamma^{\alpha}=\Delta t\left[(1-\theta) \dot{\gamma}_{t}^{\alpha}+\theta \dot{\gamma}_{t+\Delta t}^{\alpha}\right]
$$

here $\theta$ is an integration parameter ranging from 0 to 1 , while $\theta=0$ corresponds to the simple Eulerian time integration scheme. Peirce recommended a choice of $\theta$ between 0.5 and 1 [25]. The Taylor expansion of slipping rate $\dot{\gamma}^{\alpha}$ is written as

$$
\dot{\gamma}_{t+\Delta t}^{\alpha}=\dot{\gamma}_{t}^{\alpha}+\frac{\partial \dot{\gamma}^{\alpha}}{\partial \tau^{\alpha}} \Delta \tau^{\alpha}+\frac{\partial \dot{\gamma}^{\alpha}}{\partial g^{\alpha}} \Delta g^{\alpha}
$$

Therefore, the incremental expression of shear strain is

$$
\Delta \gamma^{\alpha}=\Delta t\left[\dot{\gamma}_{t}^{\alpha}+\theta \frac{\partial \dot{\gamma}^{\alpha}}{\partial \tau^{\alpha}} \Delta \tau^{\alpha}+\theta \frac{\partial \dot{\gamma}^{\alpha}}{\partial g^{\alpha}} \Delta g^{\alpha}\right]
$$

So the following formulas are obtained based on Eq. (19)

$$
\begin{array}{r}
\frac{\partial \dot{\gamma}^{\alpha}}{\partial \tau^{\alpha}}=\frac{Q_{0} p q}{k T \widehat{\tau}^{\alpha}} \dot{\gamma}_{0}^{\alpha} \exp \left\{-\frac{Q_{0}}{k T}\left[1-\left(\frac{\left|\tau^{\alpha}\right|-g^{\alpha}}{\widehat{\tau}^{\alpha}}\right)^{p}\right]^{q}\right\} \\
\cdot\left[1-\left(\frac{\left|\tau^{\alpha}\right|-g^{\alpha}}{\widehat{\tau}^{\alpha}}\right)^{p}\right]^{q-1}\left(\frac{\left|\tau^{\alpha}\right|-g^{\alpha}}{\widehat{\tau}^{\alpha}}\right)^{p-1}
\end{array}
$$


Table 1. Typical material parameters for $\mathbf{C u}$

\begin{tabular}{lll}
\hline Elastic modulus $(\mathrm{MPa})$ & Flow parameters & Hardening parameters \\
\hline$C_{11}=166,100$ & $\dot{\gamma}_{0}=10^{6} \mathrm{~s}^{-1}$ & $\lambda=0.3$ \\
$C_{12}=121,900$ & $Q_{0}=2.77 \times 10^{-19} \mathrm{~J}$ & $b^{\alpha}=0.257 \mathrm{~nm}$ \\
$C_{44}=75,600$ & $\varphi=0.7$ & $\omega_{1}=1.5, \omega_{2}=1.2$ \\
$G_{0}=49,000$ & $\widehat{\tau}_{0}=40.0 \mathrm{MPa}$ & $\omega_{\mathrm{i} 1}=1.6, \omega_{\mathrm{i} 2}=1.1$ \\
& $p=0.2, q=1.2$ & $\rho_{\mathrm{e}}^{0}=80,000 \mathrm{~mm}^{-2}, \rho_{\mathrm{s}}^{0}=80,000 \mathrm{~mm}^{-2}$ \\
& $d_{\mathrm{q}}=2.4 \mathrm{~nm}$ & $C_{\mathrm{e}}=0.5, K_{\mathrm{e}}=0.014, d_{\mathrm{e}}=1.0 \mathrm{~nm}$ \\
& $d_{\mathrm{def}}=2.5 \mathrm{~nm}$ & $C_{\mathrm{s}}=0.5, K_{\mathrm{s}}=0.028, d_{\mathrm{s}}=5.0 \mathrm{~nm}$ \\
\hline
\end{tabular}

$$
\begin{aligned}
& \frac{\partial \dot{\gamma}^{\alpha}}{\partial g^{\alpha}}=-\frac{Q_{0} p q}{k T \widehat{\tau}^{\alpha}} \dot{\gamma}_{0}^{\alpha} \exp \left\{-\frac{Q_{0}}{k T}\left[1-\left(\frac{\left|\tau^{\alpha}\right|-g^{\alpha}}{\widehat{\tau}^{\alpha}}\right)^{p}\right]^{q}\right\} \\
& \cdot\left[1-\left(\frac{\left|\tau^{\alpha}\right|-g^{\alpha}}{\widehat{\tau}^{\alpha}}\right)^{p}\right]^{q-1}\left(\frac{\left|\tau^{\alpha}\right|-g^{\alpha}}{\widehat{\tau}^{\alpha}}\right)^{p-1} \operatorname{sgn}\left(\tau^{\alpha}\right)
\end{aligned}
$$

The incremental expression of $\Delta \tau^{\alpha}$ and $\Delta g^{\alpha}$ can be derived via Eqs. (18) and (19) as follows

$$
\begin{gathered}
\Delta \tau^{\alpha}=\left[L_{i j k l} \mu_{k l}^{\alpha}+W_{i k}^{\alpha} \sigma_{j k}+W_{j k}^{\alpha} \sigma_{i k}\right] \cdot\left[\Delta \varepsilon_{i j}-\sum_{\beta} \mu_{i j}^{\beta} \Delta \gamma^{\beta}\right] \\
\Delta g^{\alpha}=\lambda G b^{\alpha} \frac{\sum_{\beta=1}^{N}\left[h^{\alpha \beta}\left(\Delta \rho_{\mathrm{e}}^{\beta}+\Delta \rho_{\mathrm{s}}^{\beta}\right)\right]+\sum_{\beta=1}^{N} i^{\alpha \beta} \Delta \rho_{\mathrm{def}}^{\beta}}{2 \sqrt{\sum_{\beta=1}^{N}\left[h^{\alpha \beta}\left(\rho_{\mathrm{e}}^{\beta}+\rho_{\mathrm{s}}^{\beta}\right)\right]+\sum_{\beta=1}^{N} i^{\alpha \beta} \rho_{\mathrm{def}}^{\beta}}}
\end{gathered}
$$

The shear strain increment $\Delta \gamma^{\alpha}$ can be calculated by Eqs. (37)-(41). Once $\Delta \gamma^{\alpha}$ is determined, the increments of other variables can be also derived.

\section{Material Parameters}

There are 12 crystallographic equivalent slip systems $\{111\}\langle 110\rangle$ in a FCC metal crystal. The dislocation density of typical fully annealed FCC pure metal is about $2 \times 10^{12} \mathrm{~m}^{-2}$ [17]. It is assumed that the initial densities of edge and screw dislocations in each slip system are the same, and the value is about $80,000 \mathrm{~mm}^{-2}$. The $\widehat{\tau}_{0}$ for unirradiated copper mainly depends on dislocations and is set as $20 \mathrm{MPa}$. For irradiated copper, besides the main radiation defect SFT, there are many other types of defects, such as voids, small clusters of self-interstitial atoms, Frank dislocation loops, precipitated phase, which would also impede the dislocation motion and result in irradiation hardening. The influence of SFT on slip resistance is considered by the evolution Eqs. (25)-(35). Besides, the influences of other defects on slip resistance also need to be considered. According to [11], the slip resistance caused by irradiation defects is assumed the same as the unirradiated lattice friction stress. Therefore, the $\widehat{\tau}_{0}$ for irradiated copper includes the influences of dislocations and irradiation defects, and its value is set as the sum of the $20 \mathrm{MPa}$ caused by dislocations and the $20 \mathrm{MPa}$ caused by irradiation effects, that is, $40 \mathrm{MPa}$.

The annihilation critical distance in Eq. (25) is $1-3 \mathrm{~nm}$ [19-21], here $d_{\mathrm{q}}=2.4 \mathrm{~nm}$. Kawasaki [26] found that the mean free path lengths of edge dislocations and screw dislocations are about $100 \mu \mathrm{m}$ and $200 \mu \mathrm{m}$, respectively. The total mean free path in Eq. (26) is the average value of the above two types. The value of $\varphi$ in Eq. (27) ranges from 0.5 to 1 , here $\varphi=0.7$ [27, 28]. The mean size of SFTs is about $2.3-2.6 \mathrm{~nm}$ [5], so $d_{\text {def }}$ is chosen as $2.5 \mathrm{~nm}$. According to the simulation and corresponding experimental results, $\omega_{\mathrm{i} 1}=1.6, \omega_{\mathrm{i} 2}=1.1$. Other material parameters can be found in [11,29], and all the parameters used are shown in Table 1. 


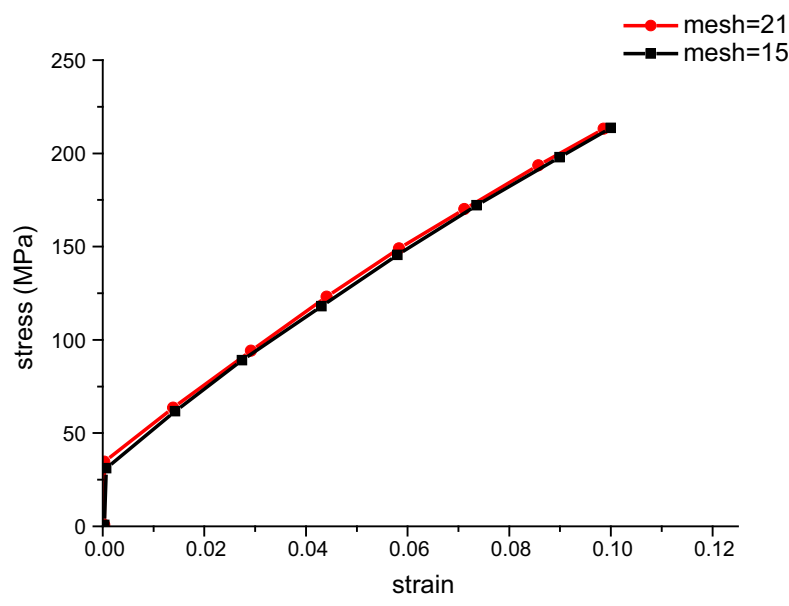

Fig. 2. Mesh independence verification of [111] orientation

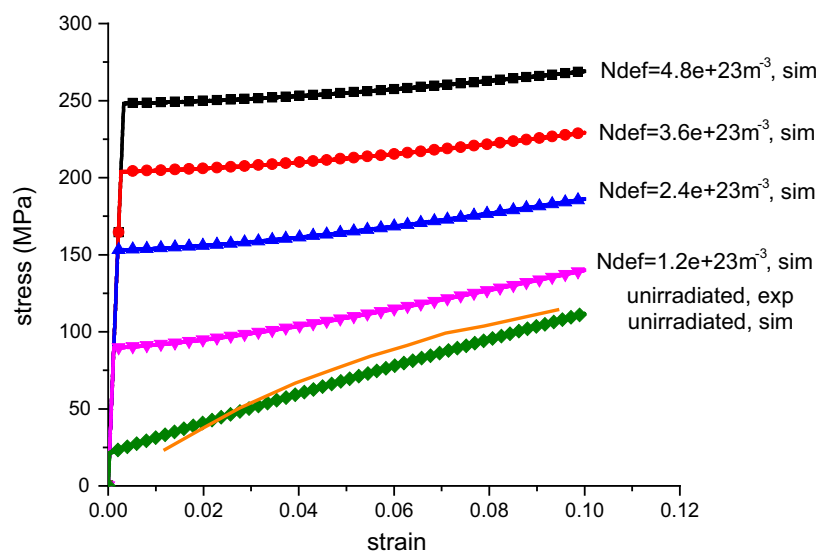

Fig. 3. Simulation and experiment [30] results of stress-strain curves in [110]

\section{Simulation Results and Discussion}

\subsection{Mechanical Behavior of Single Crystal}

According to the tensile test sample of Takeuchi experiment [30], a $\mathrm{Cu}$ single-crystal model of $22.5 \mathrm{~mm} \times 5 \mathrm{~mm} \times 2 \mathrm{~mm}$ is established. In order to approach the real uniaxial tensile process, the symmetric boundary condition is applied at one end of the model, and a displacement-controlled load is applied at the other end with a true strain rate of $3 \times 10^{-3} \mathrm{~s}^{-1}$. The testing temperature is set to be $295 \mathrm{~K}$ and the shear modulus $G=45,000 \mathrm{MPa}$ [29]. The element type is C3D8. To ensure the precision of the simulation results, the mesh independence is verified. The model is divided into 210 and 1520 grids separately, and the stress-strain curves are basically the same (Fig. 2). Consequently, the model is divided into 210 grids with less computation time.

Since the single crystal is anisotropic, the results in crystal orientations of [100], [111] and [112] are calculated with the UMAT, respectively. The stress-strain curves under different irradiation doses are shown in Figs. 3, 4 and 5.

From Figs. 3, 4 and 5, it is demonstrated that different orientations show different mechanical behaviors under uniaxial tensile simulation due to the anisotropy of single crystal. The highest yield strength occurs in [111]. It can also be concluded that the yield strength increases significantly in every orientation and grows with the increase of irradiation dose. 


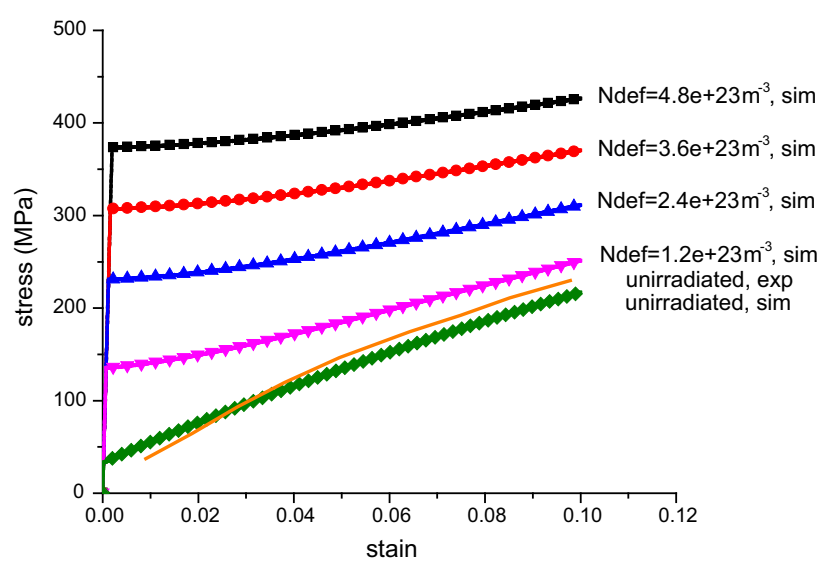

Fig. 4. Simulation and experiment [30] results of stress-strain curves in [111]

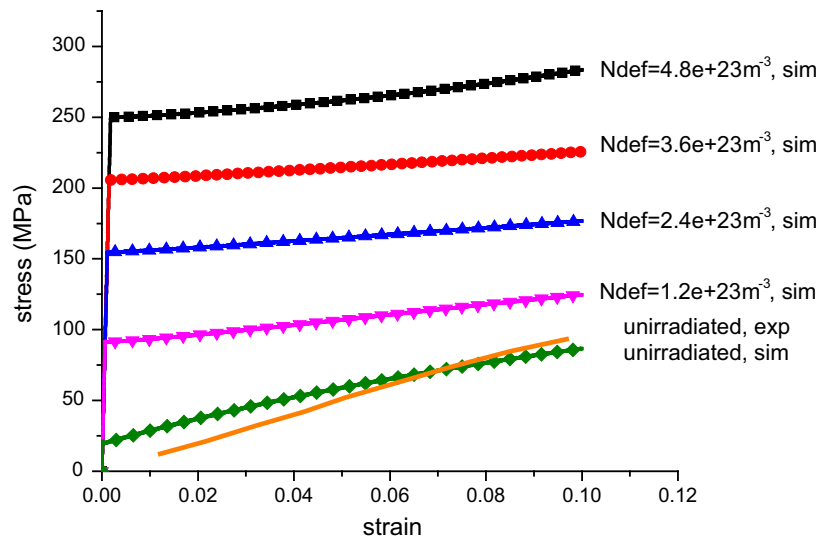

Fig. 5. Simulation and experiment [30] results of stress-strain curves in [112]

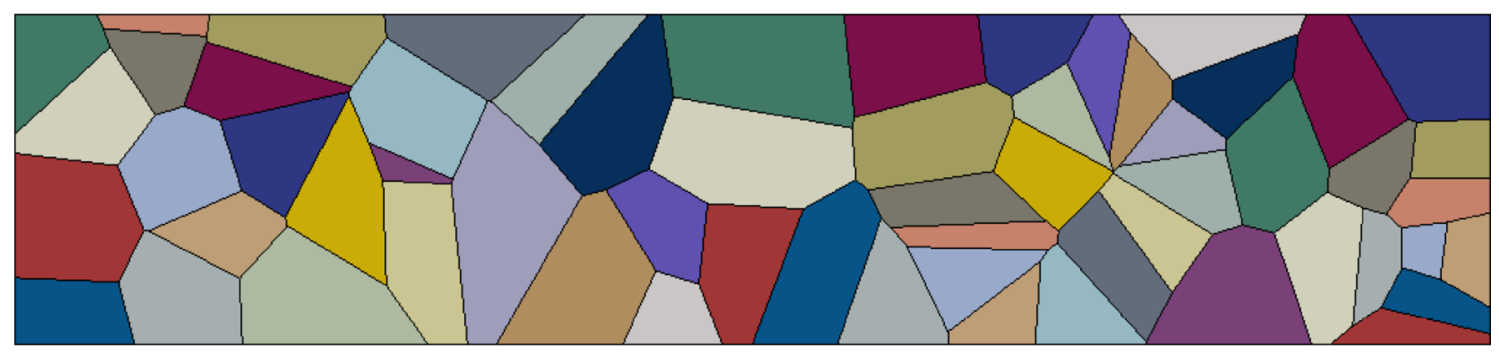

Fig. 6. OFHC Cu polycrystalline model

\subsection{Mechanical Behavior of Polycrystal}

Singh [5] conducted the irradiation experiment of OFHC copper and studied the change of the mechanical behavior. Since the polycrystal is composed of lots of small single crystal grains with different spatial orientations, the polycrystal displays macroscopic isotropy. With the aim to facilitate the analysis, the grain boundary is assumed as pure geometric plane, which can ensure the continuity of deformation and the compatibility of displacement. In the simplified grain boundary model, the dislocation motion is impeded by a large number of defects in boundaries, so the dislocation slipping mainly occurs at interior of grains [31]. Since the grains of OFHC Cu used by Singh [5] were relative large and the sample was fully annealed, the hypothesis is consistent with the actual structure. A plane strain model of polycrystal is established as shown in Fig. 6 . 
Table 2. Average size and volume density of SFTs under different values of DPA [5]

\begin{tabular}{lll}
\hline DPA & $d_{\text {def }}(\mathrm{nm})$ & $N_{\text {def }}\left(10^{23} \mathrm{~m}^{-3}\right)$ \\
\hline 0.01 & 2.3 & 2.4 \\
0.1 & 2.4 & 4.5 \\
0.2 & 2.6 & 4.5 \\
0.3 & 2.4 & 4.3 \\
\hline
\end{tabular}

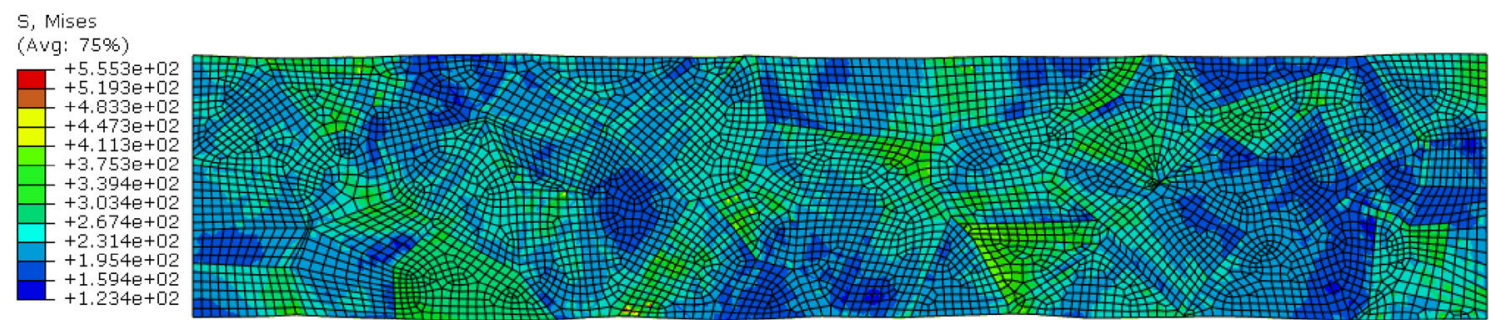

Fig. 7. Mises stress contour of uniaxial tension of polycrystalline model (MPa)

The polycrystalline model is generated by Voroni principle, and the map of 60 grains is generated in the $0.45 \mathrm{~mm} \times 0.1 \mathrm{~mm}$ plane with an average 30 -micron grain size. The average grain size is in accordance with the experimental sample.

The volume density and average size of SFTs in irradiated OFHC $\mathrm{Cu}$ are shown in Table 2. As can be seen, the volume density of SFTs increases accordingly with the increase of DPA. When DPA reaches a certain value, the volume density of SFTs is nearly saturated. Assuming that the initial density of defects in each system is the same and there are 12 slip systems in a FCC crystal, the $N_{\text {def }}^{\alpha}$ in each slip system is $2 \times 10^{22} \mathrm{~m}^{-3}$ when DPA is 0.01 , and $3.75 \times 10^{22} \mathrm{~m}^{-3}$ when DPA is 0.1 . The testing temperature is set to be $373 \mathrm{~K}$, the shear modulus $G$ decreases to $44,000 \mathrm{MPa}$ and the temperature-dependent elastic constants $C_{11}=163,800 \mathrm{MPa}, C_{12}=120,400 \mathrm{MPa}, C_{44}=73,500 \mathrm{MPa}$ are calculated by Varshni's formula [32]. Based on the experimental data for irradiated copper [1,5], the coefficients $h^{\alpha \beta}$ and $i^{\alpha \beta}$ are adjusted to reflect the influence of temperature on the evolution of dislocations and defects with $\omega_{1}=1$ and $\omega_{\mathrm{i} 1}=1.1$. Then the uniaxial tensile mechanical behaviors of non-irradiated $\mathrm{OFHC} \mathrm{Cu}$ as well as the $\mathrm{OFHC} \mathrm{Cu}$ under the conditions of $\mathrm{DPA}=0.01$ and $\mathrm{DPA}=0.1$ are simulated respectively.

Since the deformation is not uniform in the polycrystalline uniaxial tension, the stress and strain are obtained by the volume averaging method,

$$
\left\{\begin{array}{l}
\bar{\sigma}=\sum_{k=1}^{N} v^{k} \sigma^{k} \\
\bar{\varepsilon}=\sum_{k=1}^{N} v^{k} \varepsilon^{k}
\end{array}\right.
$$

here $\bar{\sigma}$ and $\bar{\varepsilon}$ are the total average stress and strain, respectively. $\sigma^{k}$ and $\varepsilon^{k}$ are the average stress and strain at integration point $\mathrm{k}$, respectively. $v^{k}$ is the volume of integration point $k$.

The stress and strain contours calculated by the uniaxial tension of polycrystalline model are shown in Figs. 7 and 8. By comparing the grain distribution of the model, it is found that the stress and strain are larger at the boundary of grains, but smaller inside the grains, which reflects that the dislocations accumulate at the grain boundary, resulting in stress concentration and non-uniform deformation.

According to the above calculation, the average stress-strain curves of uniaxial tension of polycrystalline model at different conditions are simulated. The results of three sets of random crystal orientation are shown in Fig. 9. It can be seen that the stress-strain curves are very close and have the same trend, indicating that 60 grains are sufficient to reflect the tensile properties of the material. 


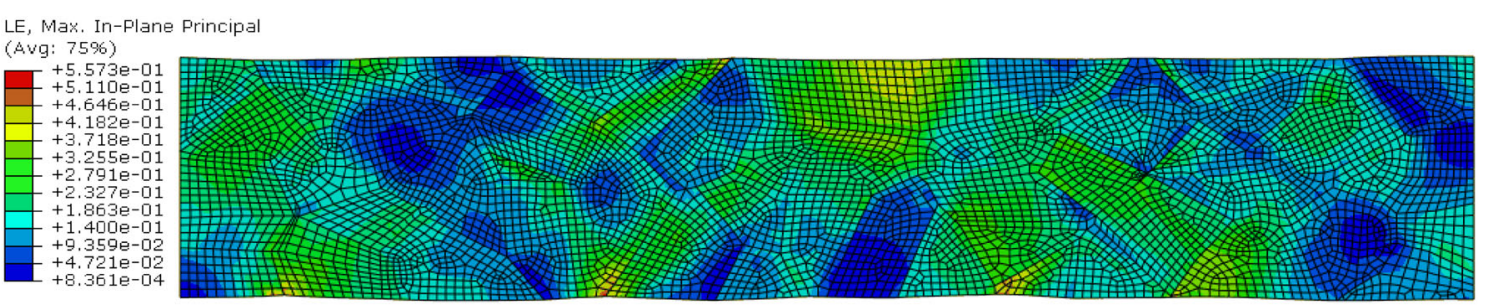

Fig. 8. The maximum principal strain contour of uniaxial tension of polycrystalline model

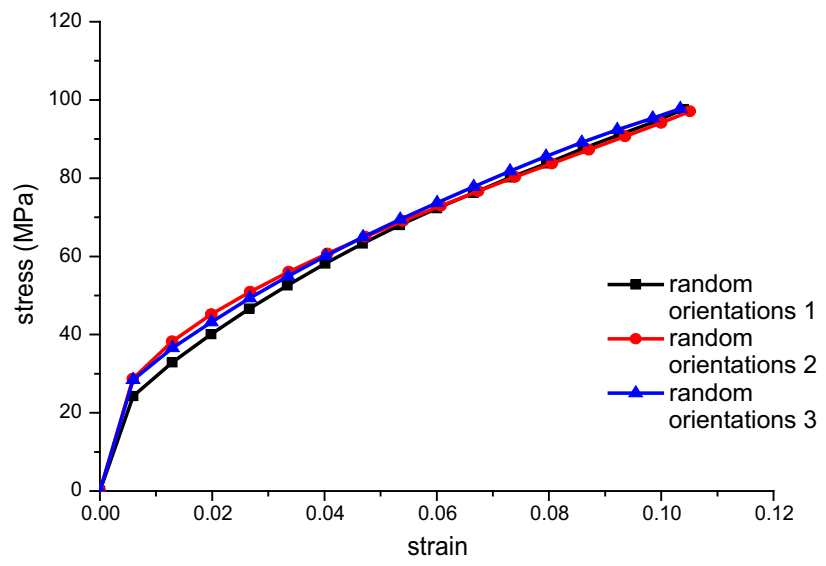

Fig. 9. Crystal orientation dependence verification with 3 sets of random orientations

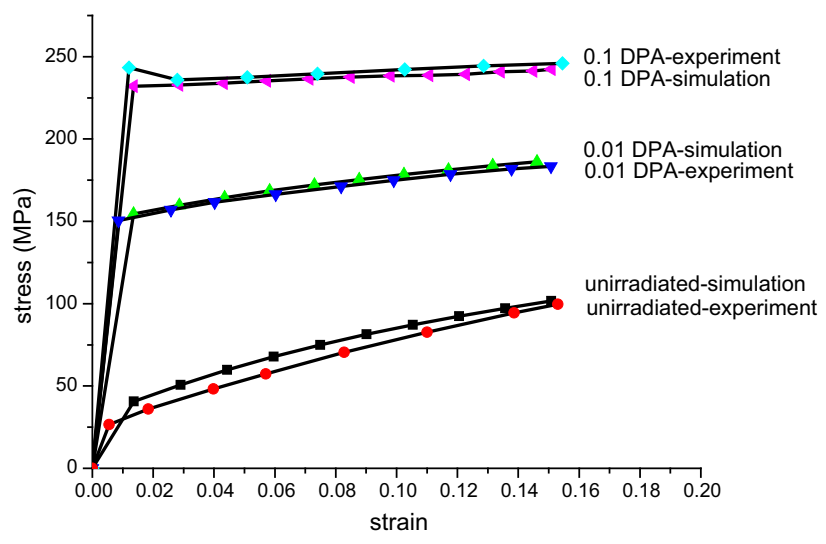

Fig. 10. Simulation and experiment [5] results of $\mathrm{OFHC} \mathrm{Cu}$

The simulation results of $\mathrm{OFHC} \mathrm{Cu}$, as shown in Fig. 10, are in good agreement with the experimental data given by Singh [5] as a whole. With the increase of irradiation dose, the strength of the material increases gradually, and the increments of the yield strength are more obvious.

\section{Conclusion}

In this paper, a crystal plasticity constitutive model with the irradiation effect is developed, which incorporates the microscopic interactions between dislocations and irradiation defects into the crystal plasticity theory. In the model, the effects of edge dislocations and screw dislocations on irradiation defects are considered, respectively. Then the model is numerically implemented and applied to simulate the uniaxial tensile deformation of single and polycrystalline copper subjected to neutron irradiation. The simulation results show that: 
(1) The constitutive model can reflect the anisotropy of single crystal and the mechanical behavior change of the irradiated material, such as the increment of yield strength.

(2) This constitutive model is able to reflect the effect of irradiation dose on the mechanical behavior of pure copper: with the increase of irradiation dose, the yield strength increases as well.

(3) For polycrystalline copper material, the simulation results are in good agreement with the experimental data.

Acknowledgements. The support of the National Natural Science Foundation of China (NSFC) under Grant No. 11202114, Beijing Higher Education Young Elite Teacher Project under Grant No. YETP0156 and Tsinghua University Initiative Scientific Research Program under Grant No. 2019Z08QCX06 are gratefully acknowledged.

Open Access This article is distributed under the terms of the Creative Commons Attribution 4.0 International License (http://creativecommons.org/licenses/by/4.0/), which permits unrestricted use, distribution, and reproduction in any medium, provided you give appropriate credit to the original author(s) and the source, provide a link to the Creative Commons license, and indicate if changes were made.

\section{References}

[1] Singh BN, Foreman AJE, Trinkaus H. Radiation hardening revisited: role of intracascade clustering. J Nucl Mater. 1997;249(2):103-15.

[2] Pokor C, Brechet Y, Dubuisson P, et al. Irradiation damage in 304 and 316 stainless steels: experimental investigation and modeling. Part II: irradiation induced hardening. J Nucl Mater. 2004;326(1):30-7.

[3] Müller GV, Gavillet D, Victoria M, et al. Post irradiation tensile properties of Mo and Mo alloys irradiated with $600 \mathrm{MeV}$ protons. J Nucl Mater. 1994;212:1283-7.

[4] Victoria M, Baluc N, Bailat C, et al. The microstructure and associated tensile properties of irradiated fcc and bcc metals. J Nucl Mater. 2000;276(1):114-22.

[5] Singh BN, Edwards DJ, Toft P. Effect of neutron irradiation and post-irradiation annealing on microstructure and mechanical properties of OFHC-copper. J Nucl Mater. 2001;299(3):205-18.

[6] Osetsky YN, Bacon DJ. Defect cluster formation in displacement cascades in copper. Nucl Instrum Methods Phys Res Sect B. 2001;180(1):85-90.

[7] Hill R. Generalized constitutive relations for incremental deformation of metal crystals by multislip. J Mech Phys Solids. 1966;14(2):95-102.

[8] Hill R. The essential structure of constitutive laws for metal composites and polycrystals. J Mech Phys Solids. 1967;15(2):79-95.

[9] Asaro RJ. Micromechanics of crystals and polycrystals. Adv Appl Mech. 1983;23:1-115.

[10] Asaro RJ. Crystal plasticity. J Appl Mech. 1983;50(4b):921-34.

[11] Cheong KS, Busso EP. Discrete dislocation density modelling of single phase FCC polycrystal aggregates. Acta Mater. 2004;52(19):5665-75.

[12] Arsenlis $\S$, A, Wirth BD, Rhee M. Dislocation density-based constitutive model for the mechanical behaviour of irradiated Cu. Philos Mag. 2004; 84(34):3617-35.

[13] Rahul De S. Multiscale modeling of irradiated polycrystalline FCC metals. Int J Solids Struct. 2014;51(2324):3919-30.

[14] Chen LR, Xiao XZ, Yu L, et al. Texture evolution and mechanical behaviour of irradiated face-centred cubic metals. Proc R Soc Math Phys Eng Sci. 2018;474(2210):20170604.

[15] Xiao X, Song D, Xue J, et al. A self-consistent plasticity theory for modeling the thermo-mechanical properties of irradiated FCC metallic polycrystals. J Mech Phys Solids. 2015;78(1):1-16.

[16] Xiao X, Song D, Xue J, et al. A size-dependent tensorial plasticity model for FCC single crystal with irradiation. Int J Plast. 2015;65:152-67.

[17] Krishna S, Zamiri A, De S. Dislocation and defect density-based micromechanical modeling of the mechanical behavior of fcc metals under neutron irradiation. Philos Mag. 2010;90(30):4013-25.

[18] Kocks WF. Thermodynamics and kinetics of slip. Progr Mater Sci. 1975;19:291.

[19] Fan H, Wang Q, Ouyang C. Comprehensive molecular dynamics simulations of the stacking fault tetrahedron interacting with a mixed dislocation at elevated temperature. J Nucl Mater. 2015;465:245-53.

[20] Fan H, Wang Q. A complete absorption mechanism of stacking fault tetrahedron by screw dislocation in copper. J Nucl Mater. 2013;441(1-3):211-5.

[21] Fan H, El-Awady JA, Wang Q. Towards further understanding of stacking fault tetrahedron absorption and defect-free channels - a molecular dynamics study. J Nucl Mater. 2015;458:176-86.

[22] Ghoniem NM, Tong SH, Singh BN, et al. On dislocation interaction with radiation-induced defect clusters and plastic flow localization in fcc metals. Philos Mag A. 2001;81(11):2743-64. 
[23] Ghoniem NM, Singh BN, Sun LZ, et al. Interaction and accumulation of glissile defect clusters near dislocations. J Nucl Mater. 2000;276(1):166-77.

[24] Hibbitt K, Sorensen, ABAQUS, Inc. Providence, RI, v6.5;2005.

[25] Peirce D, Shih CF, Needleman A. A tangent modulus method for rate dependent solids. Comput Struct. $1984 ; 18(5): 875-87$.

[26] Kawasaki Y. Correspondence between layered cell structures and slip lines in deformed copper single crystals. Jpn J Appl Phys. 1979;18:1429.

[27] Martinez E, Marian J, Arsenlis A, et al. A dislocation dynamics study of the strength of stacking fault tetrahedra. Part I: interactions with screw dislocations. Philos Mag. 2008;88(6):809-40.

[28] Martinez E, Marian J, Perlado JM. A dislocation dynamics study of the strength of stacking fault tetrahedra. Part II: interactions with mixed and edge dislocations. Philos Mag. 2008;88(6):841-63.

[29] Cheong KS, Busso EP, Arsenlis A. A study of microstructural length scale effects on the behaviour of FCC polycrystals using strain gradient concepts. Int J Plast. 2005;21(9):1797-814.

[30] Takeuchi T. Work hardening of copper single crystals with multiple glide orientations. Trans Jpn Inst Met. 1975;16(10):629-40.

[31] Borg U. A strain gradient crystal plasticity analysis of grain size effects in polycrystals. Eur J Mech A/Solids. 2007;26(2):313-24.

[32] Varshni YP. Temperature dependence of the elastic constants. Phys Rev B. 1970;2:3952-8. 